\title{
Influence of InAs Coverage on Transition of Size Distribution and Optical Properties of InAs Quantum Dots
}

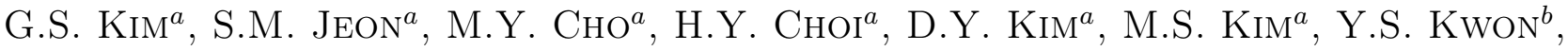 \\ J.W. CHOE ${ }^{c}$, J.S. KIM ${ }^{d}$, J.S. KIM ${ }^{e}$ AND J.Y. LEEM ${ }^{a, *}$ \\ ${ }^{a}$ Department of Nano Systems Engineering, Center for Nano Manufacturing, Inje University \\ Gimhae 621-749, Korea \\ ${ }^{b}$ ATTO Co., Ltd., Shiheung 429-849, Korea \\ ${ }^{c}$ Department of Physics, Kyung Hee University, Seoul 130-701, Korea \\ ${ }^{d}$ Department of Information Materials Science and Engineering, Chonbuk University, Jeonju 664-14, Korea \\ ${ }^{e}$ Department of Physics, Yeungnam University, Gyeongsan 712-749, Korea
}

(Received May 25, 2009; revised version April 27, 2010; in final form June 8, 2010)

\begin{abstract}
The influence of InAs coverage on the formation of self-assembled quantum dots grown by molecular-beam epitaxy was investigated by atomic force microscopy and photoluminescence measurements. As the InAs coverage increased from 2.0 to 3.0 monolayers, the quantum dot density decreased from $1.1 \times 10^{11}$ to $1.36 \times 10^{10} \mathrm{~cm}^{-2}$. This result could be attributed to the coalescence of neighboring small InAs quantum dots resulting in the formation of much larger InAs quantum dots with lower quantum dot density. Atomic force microscopy results revealed that as the InAs quantum dot coverage increased, the transition of size distribution of InAs quantum dots from single-modal to multimodal occurred. The temperature-dependent photoluminescence spectra showed that the photoluminescence spectra red shifted and the photoluminescence peak intensity decreased as the InAs coverage increased. The thermal activation energy was strongly dependent on the InAs coverage, and for InAs quantum dots with 3.0 ML thick InAs coverage, this energy was estimated to be $147 \mathrm{meV}$.
\end{abstract}

PACS numbers: 81.15.Hi, 68.37.Ps, 78.55.Cr, 78.67.Hc

\section{Introduction}

The formation of self-assembled InAs quantum dots (QDs) has been intensively studied during the last decade because of their role as model systems for strong three-dimensional carrier confinement and their possible application in novel heterostructure devices [1-3]. Various fabrication techniques have been proposed to realize a zero-dimensional structure of QDs, but most of them require complex fabrication processes. Among the QD fabrication techniques, self-assembled QD growth through the Stranski-Krastanov ( $\mathrm{S}-\mathrm{K})$ mode has thus far provided the most prominent way to fabricate optoelectronic devices such as laser diodes and light-emitting diodes [4].

Although good device performances such as high differential gain, low threshold current density, and high thermal stability are expected due to the $\delta$-like density of states of the QDs, device performance has not yet been sufficiently demonstrated because of the difficulties involved in fabricating nanometer-scale QDs of high quality and good uniformity [5-7]. In many studies, various

* corresponding author; e-mail: jyleem@inje.ac.kr growth parameters such as growth temperature, growth rate, growth interruption (GRI) time, beam-equivalent pressure (BEP), and III/V ratio have been employed to optimize the QD size and density. Generally, both the size and density of InAs QDs are functions of InAs coverage and growth temperature. As the InAs coverage increases, the QD ensemble continuously increases in size and the photoluminescence (PL) peak gradually shifts to longer wavelengths [8]. This phenomenon is clearly confirmed in the present work.

Previously, a number of research groups discussed the distinctive behavior of the PL spectra of QD ensembles with bimodal or multimodal size distributions [9]. Kim et al. [7] reported a double-peak feature in the PL spectra of in situ annealed InAs QDs, which is associated with InAs QDs with a bimodal size distribution that occurs during the annealing process. Jung et al. [10] investigated the formation characteristics of self-assembled InAs QDs with a bimodal size distribution; these characteristics are largely related to the growth temperature and InAs coverage. Some experimental evidence regarding the multimodal QD size distribution and the thermally excited carrier transfer among neighboring QDs with different sizes has been obtained [11-13]. However, the influence 
of variations in the InAs coverage on the evolution of the bimodal or multimodal QD size distribution has not yet been systematically investigated.

In this paper, we present results from atomic force microscopy (AFM) measurements of InAs QD ensembles with different InAs coverage; these results reveal some interesting features associated with the transition of size distribution of InAs QDs. The AFM results indicate an evolution of single-modal or multimodal size distribution during the variations in the InAs coverage, which shows a change in the size and density of each QD family in the ensembles.

\section{Experimental details}

All the samples used in this work were grown on silicon-doped GaAs (100) substrates by molecular beam epitaxy (MBE) using solid sources. Three different QD structures with InAs thicknesses of 2.0, 2.5, and 3.0 monolayers (MLs) were prepared to investigate the influence of InAs coverage variation on the structural and optical properties of InAs QDs. The growth rates of GaAs and InAs layers were 0.251 and $0.025 \mathrm{~nm} / \mathrm{s}$, respectively. For the growth of a $500 \mathrm{~nm}$ GaAs buffer layers, the substrate temperature was set to $560^{\circ} \mathrm{C}$, after which the substrate temperature was lowered to $460{ }^{\circ} \mathrm{C}$ for the growth of InAs QD layers. After deposition of each InAs layer, a short-period growth interruption was imposed under arsenic-rich conditions. The surface morphology of InAs QD layers without GaAs capping layers was observed by ex situ AFM. To investigate the optical characteristics through PL measurements, an identical $50 \mathrm{~nm}$ GaAs capping layer was deposited on the InAs QD layers to confine carriers. For PL measurements, the $514.5 \mathrm{~nm}$ line of an argon-ion laser was used as the excitation source to generate electron-hole pairs. The temperature-dependent PL spectra were performed in the temperature range of 20-300 K. The luminescence from the sample was focused with collection lenses, then dispersed by a monochromator and detected by a liquid-nitrogen-cooled Ge detector.

\section{Results and discussion}

Figure 1 shows AFM images of InAs QDs with different InAs coverage. For the QDs with 2.0 ML thick InAs QD coverage, we observe a single-modal size distribution of InAs QDs. Upon increasing the thickness of InAs coverage, a transition from single-modal to multimodal occurs. The highest QD density is $1.1 \times 10^{11} \mathrm{~cm}^{-2}$ for the 2.0 ML thick InAs coverage, whereas the QD density for the samples with the 2.5 and 3.0 ML thick InAs coverage decreases to $1.05 \times 10^{11}$ and $1.36 \times 10^{10} \mathrm{~cm}^{-2}$, respectively. Figure 2 shows the size distribution of InAs QDs with InAs coverages of (a) 2.0, (b) 2.5, and (c) 3.0 ML. The QDs with 2.0 ML thick InAs coverage are about $31 \mathrm{~nm}$ in size, and the distribution ratio is $99.9 \%$. For the QDs with 2.5 ML thick InAs coverage, the average size of small QDs is about $31.2 \mathrm{~nm}$ and larger QDs $71.6 \mathrm{~nm}$ in size appear, but the distribution ratio of the larger QDs is only about 5\%. This size distribution remarkably changes into a multimodal size distribution upon increasing the thickness of InAs coverage up to 3.0 ML.

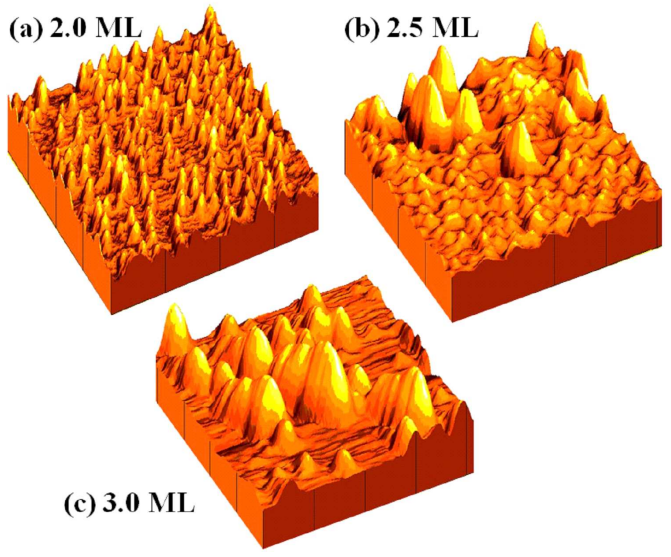

Fig. 1. AFM images of InAs QDs with coverages of (a) $2.0 \mathrm{ML}$, (b) $2.5 \mathrm{ML}$, and (c) $3.0 \mathrm{ML}$.

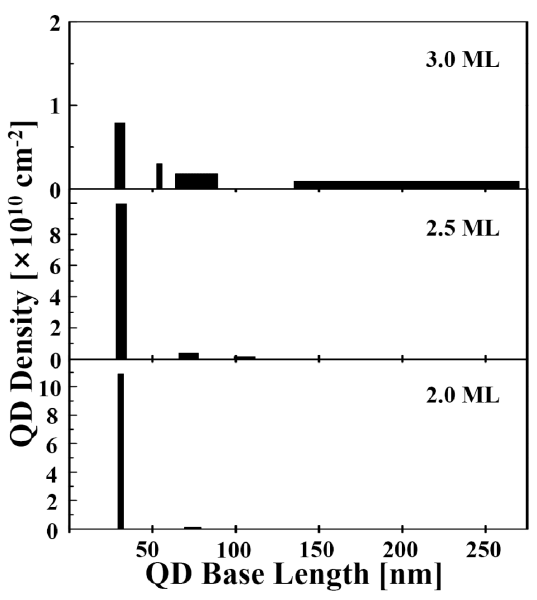

Fig. 2. Size distribution of InAs QDs with coverages of (a) $2.0 \mathrm{ML}$, (b) $2.5 \mathrm{ML}$, and (c) 3.0 ML.

For 3.0 ML thick InAs coverage, four groups of QDs are observed, whose sizes are about $30.3,54,76.3$, and $202.6 \mathrm{~nm}$. Furthermore, the distribution ratio of the four groups is about $58 \%, 22 \%, 13 \%$, and $7 \%$, respectively. The ratio of the predominant QDs to the total QD density decreases with increasing InAs coverage, being $99.06 \%$ in the $2.0 \mathrm{ML}$ sample, $95.04 \%$ in the $2.5 \mathrm{ML}$ sample, and $58.09 \%$ in the 3.0 ML sample. Coalescence with adjacent QDs or the collapse of small QDs beyond the critical QD height of $15-20 \AA$ is responsible for this effect [14]. Accordingly, an inhomogeneous size distribution becomes increasingly prominent with increasing InAs coverage, which results in the transition from a single-modal to a multimodal size distribution of InAs QDs. 
Figure 3 shows that the PL peak is red shifted with increasing InAs coverage thickness, which is responsible for the enhancement of carrier confinement that results from the lowering of the energy states. Accompanying the red shift in the PL peak is the reduction of the PL intensity, which can be directly related to the much lower QD density, as shown in the AFM images. As the InAs thickness is increased, the full width at half maximum (FWHM) of each peak is broadened from 56.8 to $68.7 \mathrm{meV}$. This is attributed to the change in the QD size distribution. The QDs with 3.0 ML thick InAs coverage have multimodal size distribution, as shown in Fig. 1, which results in the broadening of the PL peak [15].

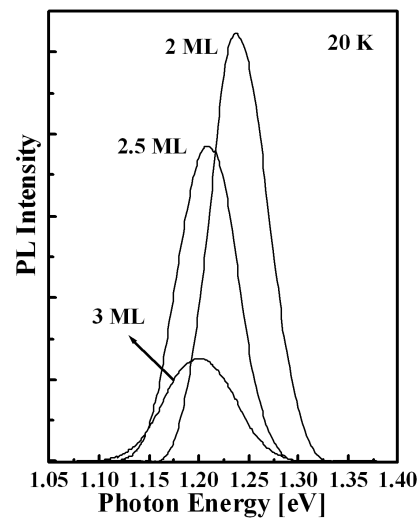

Fig. 3. $20 \mathrm{~K} \mathrm{PL} \mathrm{spectra} \mathrm{for} \mathrm{QD} \mathrm{samples} \mathrm{with} \mathrm{different}$ InAs coverages.

The temperature-dependent PL peak position and FWHM taken from the QD samples with InAs coverages of 2.0, 2.5, and 3.0 MLs are presented in Fig. 4a and b, respectively. With increasing temperature, all spectra show a typical red shift in the energy of the PL peak, which is known to be primarily due to thermally activated carrier migration from smaller to larger QDs $[12,16]$. As presented in Fig. 4b, however, the FWHM exhibits an anomalous decrease up to a temperature of $180 \mathrm{~K}$, after which it increases again with temperature. For the temperature range of $20-180 \mathrm{~K}$, the gradually thermalized carriers can repopulate nearby predominant-sized QDs. As a result of the Gaussian distribution of the QD sizes, the repopulation process will occur more frequently for the predominant QD sizes, and the FWHM of the PL spectra can decrease with increasing temperature [17]. For higher temperatures, the FWHM of the PL increases as a consequence of electron-phonon scattering, which becomes dominant. As reported in some studies conducive to the unusual temperature-dependent PL spectra in various (InGa)As QD ensembles [11, 18-22], this abnormal temperature behavior may possibly be due to thermally activated carrier redistribution between adjacent QDs with different sizes with the wetting layer serving as a carrier transfer channel.

To discuss the mechanism governing the quenching behavior of the PL spectra, the temperature dependence of
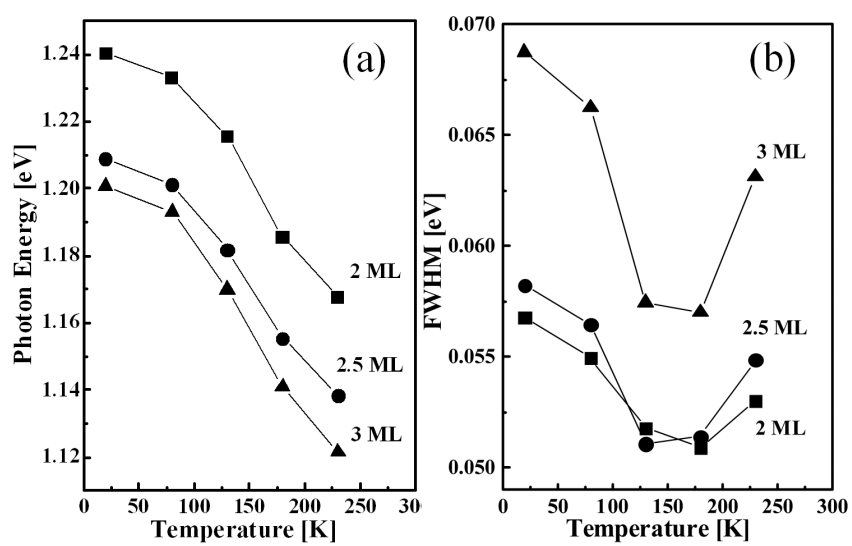

Fig. 4. (a) Peak position and (b) FWHM of temperature-dependent PL spectra for samples with InAs coverages of 2.0 and $3.0 \mathrm{ML}$, respectively.

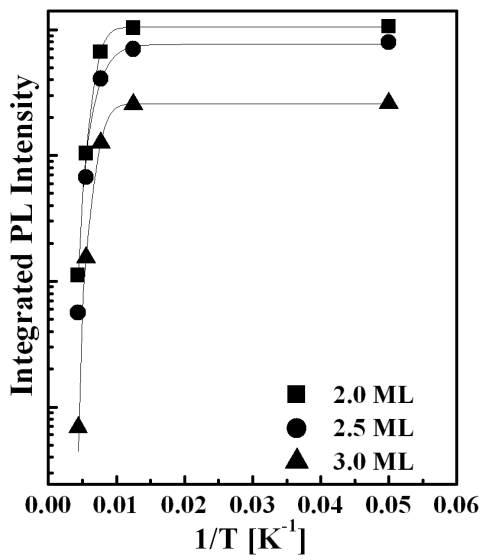

Fig. 5. Temperature dependence of integrated PL intensity for samples with different InAs coverages.

the integrated PL intensity for samples with InAs coverages of 2.0, 2.5, and 3.0 MLs are exhibited in Fig. 5. The integrated PL intensity is quite stable in the temperature range from 20 to $100 \mathrm{~K}$ and decreases dramatically for temperatures above $100 \mathrm{~K}$. The decrease in the integrated PL intensities can be related to exciton dissociation and the consequent escape of carriers from the QDs. The temperature at which exciton dissociation is observed is higher than that for quantum wells (QWs), similar to what has been reported previously [23]. With increasing temperature, the PL intensity corresponding to the confined exciton peak is given by $[24-26]: I=I_{0} /\left[1+C \exp \left(-\Delta E_{\mathrm{A}} / k_{\mathrm{B}} T\right)\right](1)$, where $I$ is the integrated PL intensity, $I_{0}$ is the PL intensity at $0 \mathrm{~K}, C$ is the ratio of the thermal escape rate to the radiation-recombination ratio, $E_{\mathrm{A}}$ is the activation energy, and $k_{\mathrm{B}}$ is the Boltzmann constant. Equation (1) assumes that the dominant mechanism for the fluctuation of the PL intensity with increasing temperature is the thermal activation of carriers from QDs to the barrier region. Larger QDs have larger activation energy due to 
the larger distance between the top of the barrier and the electronic sub-band level. Using Eq. (1), the activation energies for the samples with InAs coverages of 2.0, 2.5, and 3.0 MLs are estimated to be 116, 122, and $149 \mathrm{meV}$, respectively. The InAs QDs with $3.0 \mathrm{ML}$ thick InAs coverage have a large activation energy due to the relatively large decoupling from the ground state and the wetting layer.

\section{Conclusion}

We have investigated the influence of InAs coverage on the structural and optical properties of self-assembled QDs by performing AFM and PL measurements. Upon increasing the InAs coverage from 2.0 to 3.0 MLs, the QD density decreases from $1.1 \times 10^{11}$ to $1.36 \times 10^{10} \mathrm{~cm}^{-2}$ which is accompanied by transition from single-modal to multimodal size distribution. This result is attributed to the large size QDs that are expected to be formed from the coalescence of neighboring smaller-sized QDs or the build up at the ground state of QDs that have collapsed after exceeding the critical QD height of 15-20 $\AA$.

All the temperature-dependent PL spectra show a typical red shift of the PL peak position, which is known to be mainly due to thermally activated carrier migration from smaller to larger QDs. The FWHM exhibits an anomalous decrease up to a temperature of $180 \mathrm{~K}$, after which it increases again with temperature. This anomalous temperature behavior may possibly be due to thermally activated carrier redistribution between adjacent QDs with different sizes, with the wetting layer serving as a carrier transfer channel. Finally, the activation energy increases with increasing InAs coverage because of the large decoupling from the ground state and the wetting layer.

\section{References}

[1] D. Reuter, V. Stavarache, A.D. Wieck, M. Schwab, R. Oulton, M. Bayer, Physica E 32, 73 (2006).

[2] J.S. Kim, C.-R. Lee, I.H. Lee, J.-Y. Leem, J.S. Kim, M.-Y. Ryu, J. Appl. Phys. 102, 073501 (2007).

[3] T. Okawa, Y. Yamauchi, J. Yamamoto, J. Yoshida, K. Shimomura, J. Cryst. Growth 298, 562 (2007).

[4] J.S. Kim, J.H. Lee, S.U. Hong, W.S. Han, H.-S. Kwack, C.W. Lee, D.K. Oh, J. Appl. Phys. 94, 6603 (2003).

[5] T. Yang, M. Nishioka, Y. Arakawa, J. Cryst. Growth 310, 5469 (2008).

[6] Y. Yang, B. Jo, J. Kim, K.J. Lee, M. Ko, C.-R. Lee, J.S. Kim, D.K. Oh, J.S. Kim, J.-Y. Leem, Thin Solid Films 517, 3979 (2009).
[7] J.S. Kim, P.W. Yu, J.-Y. Leem, J.I. Lee, S.K. Noh, J.S. Kim, G.H. Kim, S.-K. Kang, S.I. Ban, S.G. Kim, Y.D. Jang, U.H. Lee, J.S. Yim, D. Lee, J. Cryst. Growth 234, 105 (2002).

[8] Y. Nakata, Y. Sugiyama, in: Self-Assembled InGaAs/ GaAs Quantum Dots Semiconductor and Semimetals, Vol. 60, Ed. M. Sugawara, Academic Press, New York 1999, p. 121.

[9] S.J. Lee, S.K. Noh, J.W. Choe, E.K. Kim, J. Cryst. Growth 267, 405 (2004).

[10] S.I. Jung, H.Y. Yeo, I. Yun, J.Y. Leem, I.K. Han, J.S. Kim, J.I. Lee, Physica E 33, 280 (2006).

[11] A. Tackeuchi, Y. Nakata, S. Muto, Y. Sugiyama, T. Inata, N. Yokoyama, Jpn. J. Appl. Phys. 34, L405 (1995).

[12] L. Brusaferri, S. Sanguinetti, E. Grilli, M. Guzzi, A. Bignazzi, F. Bogani, L. Carraresi, M. Colocci, A. Bosacchi, P. Frigeri, S. Franchi, Appl. Phys. Lett. 69, 3354 (1996).

[13] H. Lee, R. Lowe-Web, T.J. Johnson, W. Yang, P.C. Sercel, Appl. Phys. Lett. 73, 3556 (1998).

[14] B. Ilahi, L. Sfaxi, H. Maaref, G. Bremond, G. Guillot, Superlattice Microstruct. 36, 55 (2004).

[15] W. Zhou, B. Xu, H.Z. Xu, F.Q. Liu, J.B. Liang, Z.G. Wang, Z.Z. Zhu, G.H. Li, J. Electron. Mater. 28, 525 (1999).

[16] L. Kong, Z.C. Feng, Z. Wu, W. Lu, J. Appl. Phys. 106, 013512 (2009).

[17] C.Y. Lee, J.D. Song, J.M. Kim, K.S. Chang, Y.T. Lee, T.W. Kim, Mater. Res. Bull. 39, 135 (2004).

[18] H. Kissel, U. Mueller, C. Walther, W.T. Masselink, Yu.I. Mazur, G.G. Tarasov, M.P. Lisitsa, Phys. Rev. B 62, 7213 (2000).

[19] W.H. Jiang, X.L. Ye, B. Xu, H.Z. Xu, D. Ding, J.B. Liang, Z.G. Wang, J. Appl. Phys. 88, 2529 (2000).

[20] Y.C. Zhang, C.J. Huang, F.Q. Liu, B. Xu, Y.H. Chen, D. Ding, W.H. Jiang, X.L. Ye, Z.G. Wang, J. Appl. Phys. 90, 1973 (2001).

[21] S. Sanguinetti, M. Henini, M.G. Alessi, M. Capizzi, P. Frigeri, S. Franchi, Phys. Rev. B 60, 8276 (1999).

[22] H.L. Wang, D. Ning, S.L. Feng, J. Cryst. Growth 209, 630 (2000).

[23] T.-W. Kang, J.-E. Oh, J. Cryst. Growth 227-228, 1039 (2001).

[24] Z.Y. Zhang, C.L. Yang, Y.Q. Wei, X.L. Ye, P. Jin, Ch.M. Li, X.Q. Meng, B. Xu, Z.G. Wang, Solid State Commun. 126, 391 (2003).

[25] H.C. Im, J.H. Kim, D.H. Oh, T.W. Kim, K.H. Yoo, M.D. Kim, Appl. Surf. Sci. 252, 4146 (2006).

[26] K. Pterz, A. Miglo, P. Himze, F.J. Ahlers, G. Ade, I. Hapke-Wurst, U. Zeitler, R.J. Haug, Phys. Status Solidi B 224, 119 (2001). 